LA-UR -82-2676



$1 .: \div-11:-87-2676$



TITLE: THE SINGULAR PERTURBATION APPROACH TO FLAME THEORY WITH CHAIN AND COMPETING REACTIONS

AUTHOR(S): Taul $r$ pife and Bastl Nicolaenko

Submitted to: l'roceedlugs conformed on Differential liquations, Dunder 1982; lecture Notes in Mal:Lemitics 
THE SINGULAR PERTURBATION APPROACH TO FLAME THEORY

WITH CHAIN AND COMPETING REACTIONS

by

Paul C. Fife and Basil Nicolacnko

Center for Nonlinear Studies, lis B258

Los Alamos National I.ahoratory

Los Alamos, NM 87545

"i"u appriar in:

Procerenlings dinference on

IIIferential liguat ions, Dundere IOSR2;

lecelure Nolesi in Millernial ics 


\title{
THE SINGULAR PERTURBATION APPROACH TO FLAME THEORY WITH CHAIN AND COMPETING REACTIONS
}

by

Paul C. Fife ${ }^{\dagger}$ and Basil Nicolaenko

Center for Nonlinear Studies, MS B258

Los Alamos National Laboratory

Los Alamos, NM 87545

\begin{abstract}
We investigate the structure of leminar flames with two chemical reactions in the limit of high activation energy asymptolics. Depending on the specific reaction network and the other given chemical data, a wide. variety of flame configurations are possible. Here we fully explore thesc possinilities in the case of sequential and compeling reaction pairs. Our nethods are geseral enough to extend to most reaction networks wilh two or three exothermic reactions with high activation encrgy.
\end{abstract}

$f$ Depiortment of Milthemalles, Unlversity of Arizonil, Tucsun, $\Lambda \%$ 87521.

The research Hupported by NSF Grant 79-044433 and by the cinter for



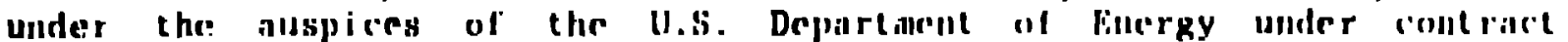

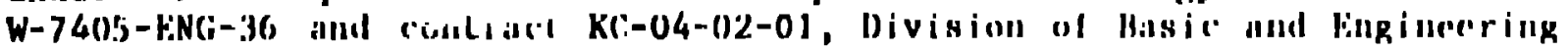
Sticuces.

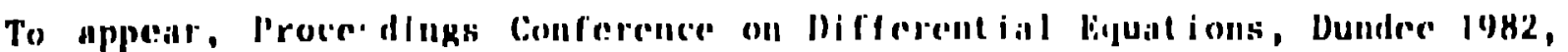
Ieceture Noles in Mathemalties, Springer-Verling, bil. 
Combustion theory provides an extremely fertile ground for asymptotic analysis. This is due in part to the fact tnit the theory combines fluid dynamics and chemical zeaction theory, both in themselves rich in difficult problems, and in part to the prevalence of small and large parameters appearing in the underlying equations.

One of the most notable large parameters expresses the fact that in combustion problems, the rates of some important chemical reactions typically depend very strongly on the temperature. In the context of the simplest cases of steady plane laminar flame theory, this fact results in a problem of the form [2]

$$
\mathrm{L} U=\mathrm{f}\left(\frac{\mathrm{U}-\mathrm{U}_{+}}{\varepsilon}, \varepsilon\right)
$$

where $\varepsilon$ is a small parameter. Here $L$ is a (vector valued) second order differential operator in the space variable $x$ whici does not depend on $\varepsilon$, and $U_{+}$is some reference vector. The solution of this problem has the following "layer" reature: the derivative $U$ ' undergoes an abrupt change with magnitude order of unity (as $\varepsilon \rightarrow 0$ ) in a small interval with length order of $c$. This incerval is called a flame layer becaluse it is here, and only here, that the sourre (reaction) term l has a sigaficantelfect. The function U, therefore, can he approximated lor small 8 by a fanction with derivalive discontinuous at one point.

This later piecewise smonth function ilsell salisties a bundiry vilue probien (Lhe "reduced problem") in which the parameter e no longer anpears: the nonlinearity has been replaced by a condition speceifying the jump in $U^{\prime}$ at its point of iliscontinuity (1.e., across the flane).

The above deseription holds when there its only one chemicat feaction in the llame process, and it is exolhermic with high activation currgy. See $|1|,|3|$ for a comprehensive treatment. When there is more than ohe reaction, the situation outlined above must be modi!ted. Depending on the speceific reaction network and the other given chenical data, a wide valledy

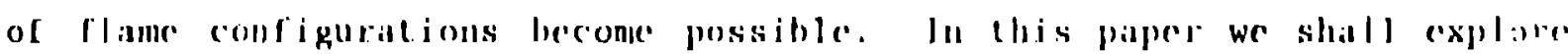
these possibilitires in the case of sequent at and competing reaction fitits. In the former case there is some overling ol our results with lhose of 
Kapila and Ludford [4]. Pairc of reactions have also been considered in [5-8]. Cases were treated in [6-8] which are in some sense limiting cases of those described here. A much fuller account of our results will be given in, In particular, the latter paper contains an extension of our methods to 1 mes with competing-fuel reaction pairs. They admit immediate extension to most reaction networks with two or three exothermic reactions with high activat ion energy.

Our analysis is largely formal, although great care is taken to justify the approximations made. They are based on the smallness of certain parameters $\varepsilon_{i}$. Often, other parameters will be assumed to be "order of unity" or " $0(1)$ ". This concept means these parameters are neither very large nor very small; otherwise, it is left without strict definition. Effectively, it means that the crror introduced by an approximation should be estimable in terms of some $\varepsilon_{i}$, or a positive power of it, multiplied by a coefficient which depends on these other parameters. The other parameters, therefore, should not be so large or small as to vitiate the accuracy of the approximation.

We begin with a description of the asymptotics and reduced problem for the standard case of a single reaction.

\section{Simple Flames}

Without giving the physical derivation, wo simply write the basic problem for laminar flanes with a single reaction $A \cdot P$. We call them simple flames. Here $\left.U=I_{Y}^{T}\right], T$ and $Y$ arc dimensionless tomperalure and concentration of reactant $A$, respectively. The problem is to delermine the "profile" $\mathrm{U}(\mathrm{x})$ and the mass flux M from the conditions

$$
\begin{aligned}
& D U^{\prime}-M\left(U^{\prime}=-w(U) K,\right. \\
& U(-\infty)=U_{-}, Y(\infty)=0 .
\end{aligned}
$$

Here $D$ is a positive definite trinsport mitrix which is linken for simplicity to be constant (Lhis is not necessiry |3]), $K$ is the veretor 
$\left[\begin{array}{c}Q \\ -1\end{array}\right]$, and $w$ is a reaction rate, which we take to be of Arrhenius type:

$$
w=B Y e^{-E / T},
$$

with $B$ and $E$ (the latter proportional to the "activation energy") positive constants. Througbout thjs paper, we assume that all elements of matrices such as $D, D^{-1}$, and vecrors $K$ are $O(1)$ (see [3] for more general circumstances).

By integrating ( $1 a)$ with use of ( $1 b)$, it is seen immediateiy that $U(\infty)$ satisfies

$$
U(\infty)=U_{+} \equiv\left[\begin{array}{c}
T_{-}+Q Y_{-} \\
n
\end{array}\right] .
$$

Thus $\mathrm{T}_{+}=\mathrm{T}_{-}+\mathrm{QY} \mathrm{Y}_{-}$. We assume

$$
\varepsilon \equiv \mathrm{T}_{+}^{2} / \mathrm{F} \ll 1
$$

and on the basis of this relation, approximale w by

$$
\omega=\frac{\eta}{\varepsilon}\left(\sum_{i}^{Y} \exp \left|\left(T-T_{+}\right) / \varepsilon\right|\right),
$$

where

$$
\eta=B \varepsilon^{2} \exp \left(-r_{i} / T_{t}\right)
$$

This approximation is obtained ly writing the exponent of (2) is in lunction


expansion in $l$ alout $l=0$. The smallness of $b$ implies (sect $l 3 \mid$ ) thitt it

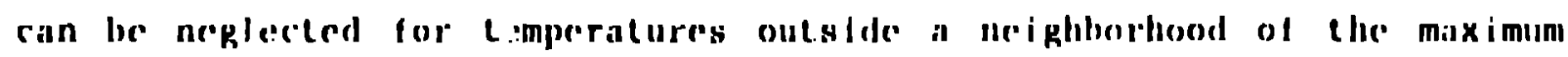
tompreriture 't 
We now perform a scale transformation $\bar{x}=x \eta^{\frac{1}{2}}, \bar{M}=M \eta^{-\frac{1}{2}} ;$ (1a) becomes (differentiation is now with respect to $\bar{x}$ )

$$
\ddot{D} \dot{\bar{M}}-\overline{\mathrm{U}}=-\frac{1}{n} \omega(\mathrm{U}) \mathrm{K}=-\frac{1}{\varepsilon} w^{*}(\mathrm{u}) \mathrm{K}
$$

where $u=\left[\begin{array}{l}t \\ y\end{array}\right]=\left(U-U_{+}\right) / \varepsilon$, and

$$
w^{*}(u)=\frac{\varepsilon}{\eta} w(u)=y e^{t}
$$

We postulate a "reduced pioblem" of the following form, for $U(\bar{x})$ (assumed contiuuous) and $\bar{M}$ :

$$
\begin{aligned}
& \text { (DE) } \quad \ddot{D U}-\bar{M} \dot{U}=0, \bar{x} \neq 0, \\
& \text { (BC) } \quad U( \pm \infty)=U_{ \pm} \\
& \text {(CC } \quad[D \dot{U}]_{0-}^{0+}=-\beta K .
\end{aligned}
$$

Here the scalar $\beta$ in the "connection condition" (9c) is to be determined a priori by a layer analysis from the function $\omega^{*}$.

By stretching variables $\xi=x i c, u=\cdots+$, and then neglecting the $t \mathrm{crm}$ formally of order $c$, we wrile (7) as

$$
1) u^{\prime \prime}+w^{\frac{\lrcorner}{*}}(u) K=0 \text {. }
$$

ihis is to be solved for $u=u(\xi)$ under llle houndary condition

$$
u(\infty)=0 \text {. }
$$

Since, from (10), $a^{\prime \prime}$ is a multiple of $D^{-1} K$ for cach $\xi$, it follows I rom (11) that u itself must be also:

$$
u(\xi)=U(\xi) D^{-1} K
$$


Using (12) in (10) and applying (11), we find a problem for $\sigma$ alone:

$$
\sigma^{\prime}+\omega^{\frac{1}{*}}\left(\sigma D^{-1} K\right)=0, \sigma(\infty)=0 .
$$

This has a first integral

$$
\left(\sigma^{\prime}\right)^{2}-\Omega(\sigma)=0,
$$

where $\Omega(\sigma)=2 \int_{\sigma}^{0} w^{*}\left(s D^{-1} K\right) d s$. Assuming now that $D^{-1} K$ has first component positive and the second negaiive, we see from ( 8$)$ that $\Omega(-\infty)$ exists, can be calculated, and represents $\left(\sigma^{\prime}(-\infty)\right)^{2}$. The matching condition between the flame layer and the "outer" solution is

$$
u^{\prime}(-\infty)=\dot{u}(0-) .
$$

From this, (12), and the fact that necessarily $U \equiv U_{+}$for $\bar{x}>0$, we may identify the constant $\beta$ in (9c) as

$$
\beta=\sigma^{\prime}(-\infty)=\left(2 \int_{-\infty}^{0} \omega^{*}\left(s D^{-1} K\right) d s\right)^{\frac{1}{2}}
$$

The specification of $\beta$ completes the definition of the reduced problem (9). What remains is to solve it. To do so, we note, by integrating ( 9 a), that

$$
\begin{aligned}
{[D \dot{U}-\bar{M} U]_{-\infty}^{0-} } & =0=D \dot{U}(0-)-\bar{M} U_{+}+\bar{M} U- \\
& =\beta K-\bar{M} Y_{-} K,
\end{aligned}
$$

hence

$$
\bar{n}_{1}=\beta / Y_{-} .
$$


With $\bar{M}$ known (9a) may now be solved for $\bar{x}<0$, with known initial conditions at $\bar{x}=0$. There is a unique sclution, and it approaches the correct limit value $U_{-}$as $\bar{x} \rightarrow-\infty$. This completes the solution of the reduced problem.

\section{The Efferts of Other Reactions on Simple Flames}

Consider a simple flame profile, as described in Sec. 1, involving a reaction term: $w_{1}(U) K_{1}$ as in (2), (5), in which the subscript "1" is appended to the quantities $E, K, \eta, E, B$, and $Y$. Thus $Y_{1}$ is the concentration of the species $A_{1}$ which is exhausted at the flame. We now perturb the problem by adding another reaction, with a concomitant second term $w_{2} K_{2}$ on the right of (la). Our main result will be a simple criterion for judging whether the second reaction constitutes a negligible perturbation of the given flame. Rescaling with use of $\eta_{1}$, we obtain the following analog of (7):

$$
\ddot{\mathrm{DU}}-\overline{\mathrm{M}} \dot{\mathrm{U}}=-\frac{1}{\varepsilon} w_{1}^{\frac{N}{*}}(\mathrm{u}) \mathrm{K}_{1}-\frac{1}{\eta_{1}} \omega_{2}(\mathrm{U}) \mathrm{K}_{2}
$$

We assume that

$$
\varepsilon_{2}=\mathrm{T}_{+}^{2} / \mathrm{E}_{2} \ll 1
$$

is also small, so that the effect of $w_{2}$ for $\bar{x}<0$ is negligible, in comparison with its effect in the flame and for $\bar{x}>0$.

There are two important cases to he considered:

(I) No reactanl for the second reaction is cxhausted in the original flame;

(II) A reactant for the second reaction is also exhausted.

Case I. This casc will apply, for example, to our sludy of sejuential rearlions in Sec. 3 . In this case, $w_{2}$ remains striclly poilive behind the 
flame, and may be represented by

$$
w_{2}=B_{2} Y_{2} e^{-E_{2} / T}
$$

We are assuming, for simplicity only, that the second $x$ action, as well as the first, is first oller: $A_{2} \rightarrow P$. As long as T deviates by amount $\circ\left(\varepsilon_{2}\right)$ from $T_{+}$, we have

$$
w_{2} \leq \mathrm{CB}_{2} e^{-\mathrm{E}_{2} / \mathrm{T}_{+}}
$$

for some constant $C=O(1)$, and the last term in (17) is bounded in magnitude by



Since the first term, die to depletion of $A_{1}$, vanishes behind the flame, the effect of the second reaction can be gauged by examinig the sciution or the initial value problem

$$
\ddot{D U}-\bar{M} \dot{U}=f(\bar{x}), \quad u(0)=u_{+}, \dot{U}(0)=0 \text {, }
$$

for vectors f with $|f| \leq r$ The representation

$$
U(\bar{x})=\int_{0}^{\bar{x}} \exp \left[\bar{M} D^{-1}(\bar{x}-s)\right] D^{-1} f(s) d s+U_{+}
$$

yields

$$
\left|U(\bar{x})-U_{+}\right| \leq \operatorname{Cqg}^{\lambda \bar{x}}
$$


for some constants $\hat{C}$ and $\lambda=O(1)$, since $\bar{M}$ and $D^{-1}$ are $O(1)$ quantities. If, therefore,

$$
q=o\left(\varepsilon_{2}\right),
$$

it follows that $\left|U(\bar{x})-U_{+}\right| \ll 1$ for $\bar{x} \geq 0$ resiricted to any interva: of length $0(1)$.

b'é wow define functions

$$
H_{i}(T)=\bar{\varepsilon}\left(\ln B_{i}-E_{i} / T\right),
$$

where

$$
\bar{\varepsilon}=\mathrm{T}_{+}^{2} / \operatorname{Min}\left(\mathrm{E}_{1}, \mathrm{E}_{2}\right)
$$

It is easily seen that $H_{1}{ }^{\prime}(T)$ is at most $O(1)$ for each $i$, and $H_{k}{ }^{\prime}\left(T_{+}\right)=1$, where $k$ is such that $E_{k}=\operatorname{Max}\left[E_{1}, E_{2}\right]$. Suppose, now, that

$$
H_{2}\left(T_{+}\right)-H_{1}\left(T_{+}\right)=-v<0 \text {. }
$$

then from (18),

$$
q=\frac{c}{\varepsilon_{1}{ }^{2}} e^{-v / \bar{\varepsilon}} \leq C \iota^{-2} e^{-\nu / \bar{\varepsilon}}=o\left(\varepsilon_{2}\right),
$$

if ( $\sim r$ example) $\nu$ is an $0(1)$ quantity.

Our conclusion is that (21) is a sufficient condition for $w_{2}$ to have a negligible effect on the original simpie flame in regions a distance $0(1)$ from the flame (actually, it is clear that much larger reginns could be allowed).

Case II. In this case, we assume for simplicity that $Y_{2}=Y_{1}=Y$. A prime example will be the branching network in Sec. 4. The iast reaction 
rate in (17) becones

$$
\begin{aligned}
\frac{1}{\eta_{1}} w_{2} & =\frac{1}{\eta_{1}} B_{2} Y e^{-E_{2} / T} \\
& \cong \frac{\varepsilon_{1}}{\eta_{1}} B_{2} e^{-E_{2} / T}+y e^{Y t} \\
& =\frac{1}{\varepsilon_{1}} r 2^{*(u)},
\end{aligned}
$$

where we have set

$$
\gamma=E_{2} / E_{1}, "{ }_{2} *=y e^{\gamma t} .
$$

and

$$
r=B_{2} e^{-E_{2} / T}+/ B_{1} e^{-E_{1} / T}+
$$

We assume $Y=U(1)$ for this exposition. Since $Y$ is depleted, $U=$ const $=$ $U_{+}$for $\bar{x}>0$, so the stretched variable $u=\left(U-L_{+}\right) / \varepsilon_{1}$ satisfies the iimit condition $u(\infty)=0$. The Jayel problem is found from (17), (22), and issumes the form

$$
\begin{aligned}
& D u^{\prime}+w_{1} \dot{x}(u) K_{1}+r w_{2} *(u) K_{2}=0, \\
& u(\infty)=0 .
\end{aligned}
$$

Reasoning in the sime minner as before, we see any solution must be of the form

$$
n(\xi)=-\sigma_{1}(\xi) D^{-1} K_{1}-\sigma_{2}\left(\xi_{1}\right) U^{-1} K_{2}
$$


for some scalars $\sigma_{j}$. Sulstituting into (25), we obtain the problem

$$
\begin{aligned}
& \sigma_{1}{ }^{\prime}-\omega_{1}{ }^{*}\left(-\Sigma \sigma_{j} D^{-1} K_{i}\right)=0, \\
& \sigma_{2}{ }^{\prime}-r \omega_{2}{ }^{*}\left(-\Sigma \sigma_{i} D^{-i} K_{i}\right)=0, \\
& \sigma_{1}(\infty)=0 .
\end{aligned}
$$

The foliowing result will be useful to us later, as well as in the present context.

Lemma. Assume the vectors $D^{-1} K_{i}$ have positive first and negative second components. Then the problem (27) has, for each $r>0$, a unique (except for translation of the inojependent variable $\xi$ ) positive solution $\sigma_{i}(\xi)$. It. satisfies $-\infty<\sigma_{i}{ }^{\prime}(-\infty)<0$. The quantities $-a_{i}=\sigma_{j}{ }^{\prime}(-\infty)$ depend contiruously on $r$, and satisfy

$$
\lim _{r \downarrow 0} \frac{a_{2}}{a_{1}}=0 \quad ; \quad \lim _{r \uparrow \infty} \frac{a_{2}}{a_{1}}=\infty
$$

Proof: The equation $(21 a, b)$ miay bu writlin as a lirst order fystem

$$
\begin{aligned}
& \sigma_{1}^{\prime}=-p_{1}, \\
& p_{1}^{\prime}=-w_{1} *, \\
& \sigma_{2}^{\prime}=-p_{2}, \\
& p_{2}^{\prime}=-r w_{2} *
\end{aligned}
$$

The object is to find a trijectory approaching the criitical point o is $\zeta, \infty$ from the positive quadrant $\mathrm{p}=\left\{\mathrm{o}_{i}, 0, \mathrm{p}_{i}=0\right\}$. It may be checked 
that the stable manifold is one dimensional and one branch of it indeed approaches 0 from $P$. We follow that branch backward: in terms of $s=-\xi$, with "." = " $\frac{d}{d s} "$, (29) becomes

$$
\begin{aligned}
& \dot{\sigma}_{1}=p_{i} \\
& \dot{p}_{1}=w_{1} * \\
& \dot{\sigma}_{2}=p_{2} \\
& \dot{p}_{2}=r w_{2}^{*} .
\end{aligned}
$$

i.et the posiljue constants $\beta$ be defined by

$$
\mathrm{I}^{-1} \mathrm{k}_{\mathrm{i}}=\left[\begin{array}{c}
\beta_{1 \mathrm{j}} \\
-\beta_{2 \mathrm{i}}
\end{array}\right] \text {. }
$$

Then for $\therefore=\left[\begin{array}{l}\sigma_{1} \\ v_{1} \\ a_{2} \\ r_{2}\end{array}\right]$ p',

$$
y=2 \beta_{2 i} \sigma_{i} \geqslant 0
$$

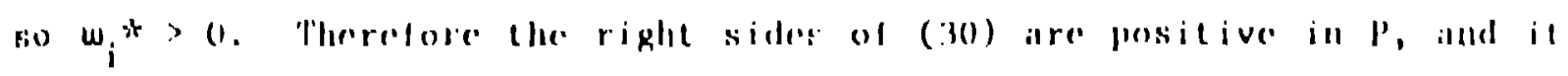

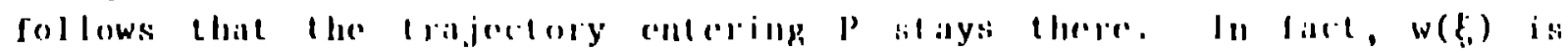


monotone increasing, and from (30), (31), we have, for some constants $c_{i}, c_{i}$

$$
\begin{aligned}
\sigma_{i} & \geq c_{1}(s-1) \\
y & \leq c_{2}\left(\sigma_{1}+\sigma_{2} ;,\right. \\
t & \leq-c_{3}\left(\sigma_{1}+\sigma_{2}\right), \\
\omega_{1} \dot{*} & \leq c_{2}\left(\sigma_{1}+\sigma_{2}\right) e^{-c_{3}\left(\sigma_{1}+\sigma_{2}\right)} \\
& \leq c_{4} e^{-c_{5}\left(\sigma_{1}+\sigma_{2}\right)} \\
& \leq c_{5} c^{-c_{6} s} .
\end{aligned}
$$

ilence from (30) agaitu,



Similar reasonillg shows

$$
P_{2} \cdot c_{1} \cdot
$$

therefore $p_{i}(s)$ approisch linile limits it $=P_{i}(\infty)$. This proves the lirtil



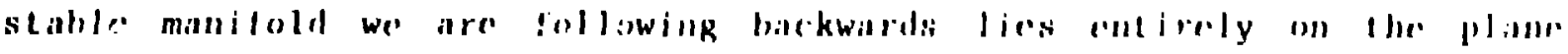

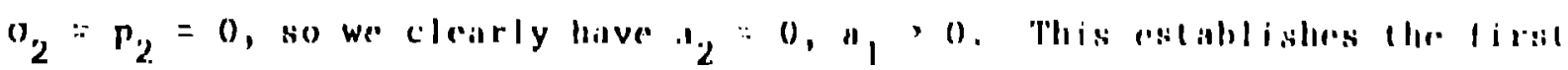

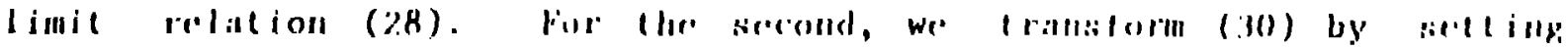




$$
\begin{aligned}
& s=\bar{s}^{-\frac{3}{2}}, p_{i}=\bar{p}_{i} r^{\frac{1}{2}}, \sigma_{i}=\bar{\sigma}_{i} \text {, then } \\
& \dot{\sigma}_{1}=\bar{p}_{1}, \\
& \dot{p}_{1}=\frac{1}{r} w_{1} *\left(\bar{\sigma}_{1}, \bar{\sigma}_{2}\right), \\
& \dot{\sigma}_{2}=\bar{p}_{2}, \\
& \dot{p}_{2}=w_{2} * .
\end{aligned}
$$

As $r+\infty, \frac{1}{r}+0$ and we use the argument symmetric to the above: it follows that $\bar{p}_{1}(\infty)+0$, hence $P_{1}(\infty)=r^{\frac{1}{2}} \bar{p}_{1}(\infty)+0$. Ihus $a_{1}+0, a_{2}=0$. This gives u: the serond of $(28)$, and completeri the prool.

firom (26), we now hilve

$$
m 1^{\prime}(-\infty)=i_{1} k_{1}+n_{1} k_{2}
$$

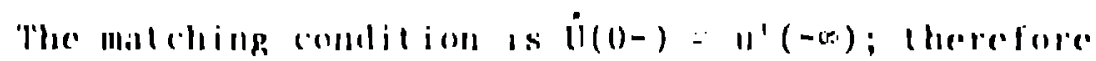

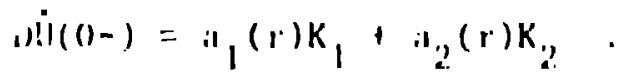

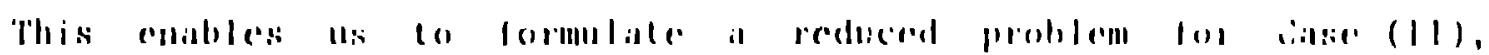
intilogous (o (9):

$$
\begin{aligned}
& \ddot{n}-\ddot{M i}: 0, x \neq 0 \text {; } \\
& 11(1(n): 11, \text {; }
\end{aligned}
$$


and (32). The solution is obtained as follcws: from (33a) we have $\operatorname{DU}\left(0^{-}\right)-\bar{M}\left(U_{+}-U_{-}\right)=0$; then from (32) and this,

$$
U_{+}-U_{-}=\frac{a_{1}(r)}{\bar{M}} K_{1}+\frac{a_{2}(r)}{\bar{M}} K_{2} \equiv \alpha_{1} K_{1}+\alpha_{2} K_{2}
$$

There are actually two conceivable forumations of the problem here:

(a) Given $U_{+}$, determine $\bar{M}, r$, and $U(\bar{x})$;

(b) Given $r$, determine $\bar{M}, U_{+}$, and $U(\bar{x})$.

of course, either case ignores the fact that $r$ and $T_{+}$are also relaled through (24), and so (24) will have to serve as a consistency check.

Both problems are solvable. For example in (a), $\alpha_{i}>0$ will be given, so we rirst determine $r$ from the condition

$$
\frac{a_{2}(r)}{a_{1}(r)}=\frac{\alpha_{2}}{\alpha_{1}}
$$

By the lemma (in particular (28)), lhere exists a value of r which satisfies this relition. It is not clear at this point whether $r$ is;


We now return to the functions $H_{i}(.9)$, and ruppose that (21) hold:i. It follows from (24) that $\ddot{i}$ ln $r=-v$, so that

$$
r=r^{-1) / \ddot{E}} \& 1
$$


moblem (13), (32) is identical to (9), and lt is casty to sece that the solution deprends continuously on ng. Hence it is justified lo megloct renction ( $i$ i).

Our conclusion, then, is the same as in Cane I: (:I) in the crilerion



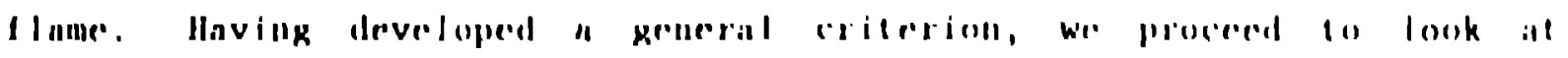
aprecilic networkw. 


\section{Reactions in Series}

We now consider a combustion process involving a pair of reactions in series:
(i)
(ii)
$A \stackrel{B}{\longrightarrow} \longrightarrow P$

both exothernic wilh high activation energy. The unburned state, in the

form $U_{-}=\left[\begin{array}{c}T_{-} \\ Y_{A_{-}} \\ Y_{B_{-}}\end{array}\right]$is prescribed. To conceptualize the possible flame struc-

Lures, we first follow the approach of Sec. 2: we imagine a simple flane resulting from only one of the reactions, the oither being artificially suppressed. We then ask whether the critericon is met, whereby tie other reaction, if no longer suppressed, would bare a negligible effect. If so, then that simple flame is a realjable strurture. After this i:i done, we investigate possible nonsimple flames.

There are iour basic simple flames:

lil, produced by reaction (i) only, converting the given unburned stille 16) a paltially burned one:

$$
U_{-} \cdot U_{1}=\left[\begin{array}{c}
\mathrm{T}_{1} \\
0 \\
\mathrm{Y}+\mathrm{Y}^{\mathrm{B}} \\
\mathrm{H}_{-}
\end{array}\right]
$$

lirom (3) it is seen that $r_{1}: Q_{1} Y_{A-}+T_{-}$.

H2, produced hy ( $i i)$ only:

$$
\mathrm{u}_{-} \cdot \mathrm{U}_{2}\left[\begin{array}{c}
\mathrm{r} \\
\mathrm{r}_{2} \\
\mathrm{\Lambda}_{-} \\
0
\end{array}\right]
$$


with $\mathrm{T}_{2}=\mathrm{T}_{-}+\mathrm{Q}_{2} \mathrm{Y}_{\mathrm{B}_{-}}$.

F12, produced by (ii) acting on the product (final state) of F1:

$$
\mathrm{U}_{1} \rightarrow \mathrm{U}_{+} \equiv\left[\begin{array}{l}
\mathrm{T}_{+} \\
0 \\
0
\end{array}\right]
$$

where $\mathrm{T}_{+}=\mathrm{T}_{-}+\mathrm{Q}_{1} \mathrm{Y}_{\mathrm{A}-}+\mathrm{Q}_{2}\left(\mathrm{Y}_{\mathrm{A}-}+\mathrm{Y}_{\mathrm{B}-}\right)$.

F21, acting on the product of $F 2$; here the reaction is of the type $A+P$ with rate identical to that oi $(i)$, but with heat release equal to the sum $Q_{1}+Q_{2}$. One can think of this flame as converting $A$ to $B$ by reaction ( $i$ ), and $B$ thereupon being almost. immediately converted to $P$ by a faster reaction ( $i i)$.

We again define functions $H_{i}(T)$ according to (19), with $\bar{l}$ given by (20). We assume that $T_{-}, T_{1}, T_{2}$, and $T_{+}$are all of the same order of magnitude, and $T_{1}-T_{-}, T_{2}-T_{-}, T_{+}-T_{1}, T_{+}-T_{2}$, all are $0(1)$ quantitie's. The temperature scale is adjustad so that. $\mathrm{T}_{+}-\mathrm{I}_{-}=1$,

We consider the consequeneres of various inequalities involving the functions $\|_{i}$. When such an incquality is wrillen, it will be assuned that the difference of the two quintities concerned is $0(1)$. This is the typical case, becaluse the range of at least one of the lunctions $H_{i}$ is of order unity.

Intmediate conserquencess of sere. 2 are:

1) If $H_{2}\left(T_{1}\right)<H_{1}\left(T_{1}\right)$, then $F 1$ is reillizillle.

2) II $H_{1}\left(T_{2}\right)<H_{2}\left(T_{2}\right)$, then $\mathrm{H}_{2}$ is .

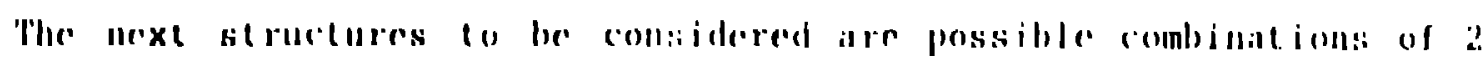

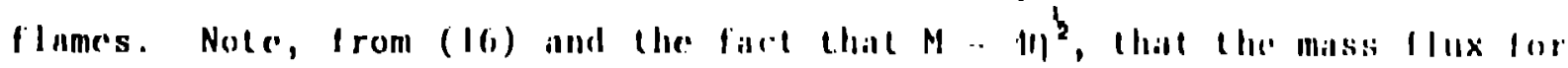
fil is

$$
M_{1}=\left(C_{1} H_{1} c_{1}^{2}\left({ }_{1}\right)\left(-N_{1} / T_{1}\right)\right.
$$


where

$$
\varepsilon_{1}=\mathrm{T}_{1}^{2} / \mathrm{E}_{1}
$$

and $C_{1}$ is some corstant, $C_{1}=0(1)$. Hence,

$$
\left.\bar{\varepsilon} \ln M_{1}=\bar{\varepsilon}\left(\ln B_{1}-E_{1} / T_{1}+2 \ln \varepsilon_{1}\right)\right)+0(\bar{\varepsilon})
$$

But $\left|\ln \varepsilon_{1}\right|=\ln \frac{1}{\varepsilon_{1}} \leq \ln 1 / \bar{\varepsilon}$

$$
\bar{\varepsilon} \ln \mathrm{N}_{1}=\mathrm{H}_{1}\left(\Gamma_{1}\right)+0(\bar{\varepsilon} \ln 1 / \bar{i})
$$

lu a similar manner,

$$
\begin{aligned}
& \bar{i} \ln \mathrm{M}_{2}=\mathrm{H}_{2}\left(\mathrm{~T}_{2}\right)+o(1), \\
& \bar{i} \ln \mathrm{M}_{12}=\mathrm{H}_{2}\left(\mathrm{I}{ }_{+}\right)+o(1), \\
& \bar{i} \ln M_{21}=H_{1}\left(\mathrm{I}_{+}\right)+o(1) .
\end{aligned}
$$

It torns out thit the mas: lluxes of these foul sinple flatues are orelereal

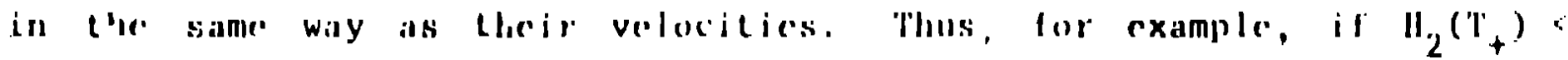
$\|_{1}\left(T_{1}\right)$, then Fl2, buile on the products ol Fl, is stower thin fil itsell, allet so one cian conceive of the two thames hoth existing, hue the distince between lhem ever increasing. Ihe flame fil precentess, and is faster lhan, Fi2. Wr call the Lotal configuilion lil, I2:

3) $\quad 11 \mathrm{HI}_{2}\left(\mathrm{r}_{1}\right)<\mathrm{H}_{1}\left(\mathrm{l} \mathrm{l}_{1}\right)$, then fil, 12 is realizible. 
Similarly:

4) If $\mathrm{H}_{1}\left(\mathrm{~T}_{+}\right)<\mathrm{H}_{2}\left(\mathrm{~T}_{2}\right)$, then $\mathrm{F} 2,12$ is.

The most interesting flames, however, are seen when neither 3) nor 4) hol. This last case is split inio two subcases: $H_{1}\left(T_{1}\right)<H_{2}\left(T_{+}\right)<$ $H_{1}\left(T_{+}\right)$, and $H_{2}\left(T_{2}\right)<H_{1}\left(T_{+}\right)<H_{2}\left(T_{+}\right)$. It can he easily checked that these two are exclusive and, excluding cases when equality holds somewhere, exhaust the complement of 3 ) and 4). Since 3) and 4) do not hold, the diverging configurations $F 1,12$ and $F 2,21$ are not possible. Indeed if such a combination of flames like F1, 12 existed at one time, then the rear flame F12 would approach the forward flames F1. A: it does so, its eflect is to heat up the forward one. As this happens, the spect! of the forward increases (this is feasible because our expression for $M$, as in (35), is an increasing function of $T$ ). The question now is whether this heating from the rear is sufficient to raise the velocity of the forward pirt to cqual that of the trailing $F 12$. Of course, the temperature of Fl cimnot by this mechanism be raised above ' $T$, which is the tenperatlere of Fl2. And it seems reasonable that the equality of speeds will be attained at it temperature $T_{0}$ such that $I_{1}\left(T_{0}\right)=H_{2}\left(T_{+}\right)$. Therefore our conjecture (to he verified) is that such a Landem configuration, with fle (ahead) and lile (behind) traveling at a fixed distance apart, is realizalle if there is a ' $\mathrm{f}_{0}$ satistying the above equation. But this is true precisely in the first subcase. We call this Landem llame fl-12.

5) If $\mathrm{H}_{1}\left(\mathrm{~T}_{1}\right)<\mathrm{H}_{2}\left(\mathrm{~T}_{+}\right)<\mathrm{H}_{1}\left(\mathrm{~T}_{+}\right)$, then $\mathrm{l} 1-12$ is reis izanble.

Similarly,

6) If $\mathrm{H}_{2}\left(\mathrm{~T}_{2}\right)<\mathrm{H}_{1}\left(\mathrm{~T}_{+}\right)<\mathrm{H}_{2}\left(\mathrm{~T}_{+}\right)$, then $\mathrm{r} 2-2 \mathrm{is}$ realizable.

1 shombl again be emphisized that at this point, (b) and (b) are just conjecetures. However, they can be verilied in the following manner. It a tandean structure exists, it should correspond lo the solution al i reduced 
problsm in the spirit of (9), and this reduced problem should be uniquely solvable.

This is, in fact, the case. For F1-12, for exanple, the reduced problem takes the form

$$
\begin{aligned}
& \ddot{D U}-\bar{M} \dot{U}=0, \bar{x} \neq 0, \bar{x} \neq 2 ; \\
& U( \pm \infty)=U_{ \pm}, \\
& {[D \dot{U}]_{0-}^{0+}=-\beta_{1} K_{1},[D \dot{U}]_{l-}^{l+}=-\beta_{2} K .}
\end{aligned}
$$

Here $\beta_{2}, U_{ \pm}$, and $T(0)=T_{0}$ consticute the given data; the proble'll is then Lo determine $\beta_{1}, \ell$, and $U(\bar{x})$. The problem has a unique solution. The constant $\beta_{2}$ is known by a layel analysis very like that in Sec. 2. However, the coefficient. $\beta_{1}$ should also be involved with t liyer analysis. Aud incleed such an analysis can be performerl; il yields a functional relation

$$
H_{1}=F(r, i(0+1))
$$

where $r$ is given hy (24) with $T_{+}$in the denominutor repliced by $T_{0}$. This relation can be inverled to yield

$$
r=R\left(\beta_{1}, \dot{u}(0+)\right)
$$

The proper consigtency check on the elbuve reduced problem, lhen, is (1) solve il, and lind $A_{1}$ and $i(1+)$ : (2) determine $r$ rom $\left.! 36\right)$; a this $r$ saltisfies (24) ('T+ in denominaler replaced by $T_{0}$ ). Wr rewrile (a/4) ill the lorm

$$
\text { i: } \ell=r=H_{2}\left(T_{1}\right)-H_{1}\left(T_{n}\right) \text {. }
$$


Now $T_{0}$ was chosen to make the right side vanish; but if we now reason that this is only the first approximation to the true value of $T_{0}$, then (37) can be used to obtain a corrected value for $T_{0}$. Since $H_{1}{ }^{\prime}\left(T_{0}\right)=0(1)$, and $r=O(1)$, it turns out that the correction will only be $0(\bar{\varepsilon})$, so the original value of $T_{0}$ will be correct within our general range of accuracy. The details of the tandem analysis can be found in [3].

In sumary, the possibility of the various flame types 1,2 , etc., can be found by testing each of the cases 1) - 6) in turn. Cases 3) - 6) arc mutually exclusive, but any of the latter could hold in conjunction with 1 ) or 2). There result nonunique flame structures; which one is realized in a given ouservatinn would depend on how and to what extent the gas mixture is ignited. $A$ ? $e$ of nonuniqueness is the case when $T_{1}<T_{2}$, and

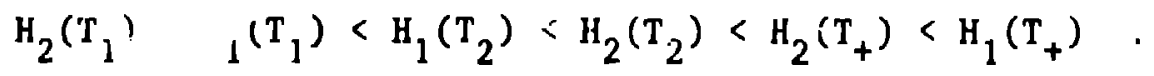

In this rase, types 1,2 , and $1-12$ are all realizable.

\section{Reactions in Parallel}

The combustion process now allows the reactant $A$ to be consumed in lwo competing fashious:

(i)

A

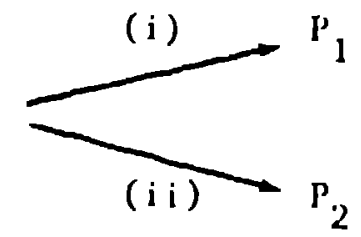

Again, each $r$ ction is exolhermic with high activiltion encrgy, an! the unhurned state $U(-\infty)=U_{-}=\left[\begin{array}{l}T_{-} \\ Y_{-}\end{array}\right]$is prescribed (here $Y$ repressents the dimensionless concent ration of $A$ ).

There are oaly two conceivable simple flames: fil and F2, with fillal temperatures $T_{1}=T_{-}+Q_{1} Y_{-}$and $\mathrm{I}_{2}=T_{-}+Q_{2} Y_{-}$, respectively. for the sake of definiteness, we assune $Q_{1}<Q_{2}$, hence

$$
\mathrm{T}_{1}<\mathrm{T}_{2}
$$


Temperature is scaled so that $\mathrm{T}_{2}-\mathrm{T}_{-}=1$. Ass re $\mathrm{T}_{2}-\mathrm{T}_{1}, \mathrm{~T}_{1}-\mathrm{T}_{-}=0$ (1). We define $h_{i}$ again by (19) and (20) with $T_{+}$replaced by $T_{2}$. According to the criterion in Sec. $2, F 1$ is realizable if $H_{1}\left(T_{1}\right)>H_{2}\left(T_{1}\right)$, and $F 2$ is if $\mathrm{H}_{2}\left(\mathrm{~T}_{2}\right)>\mathrm{H}_{1}\left(\mathrm{~T}_{2}\right)$. Four cases therefore arise:
(a) $\mathrm{H}_{1}\left(\mathrm{~T}_{i}\right)<\mathrm{H}_{2}\left(\mathrm{l}_{i}\right), i=1,2$,
(b) $\mathrm{H}_{1}\left(\mathrm{~T}_{1}\right)>\mathrm{H}_{2}\left(\mathrm{~T}_{\mathrm{i}}\right), \mathrm{i}=1,2$,
(c) $\mathrm{H}_{1}\left(\mathrm{~T}_{1}\right)>\mathrm{H}_{2}\left(\mathrm{~T}_{1}\right), \mathrm{H}_{1}\left(\mathrm{~T}_{2}\right)<\mathrm{H}_{2}\left(\mathrm{~T}_{2}\right)$,
(d) $\mathrm{H}_{1}\left(\mathrm{~T}_{1}\right)<\mathrm{H}_{2}\left(\mathrm{~T}_{1}\right), \mathrm{H}_{1}\left(\mathrm{~T}_{2}\right)>\mathrm{H}_{2}\left(\mathrm{~T}_{2}\right)$.

The cases are depicted by these four sample graphs of functions $H_{j}$ (dotted lines represent $H_{1}(T)$, dashed lines $H_{2}(T)$ ).

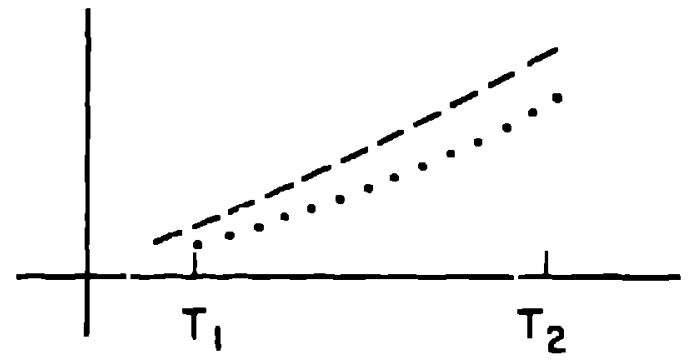

(a)

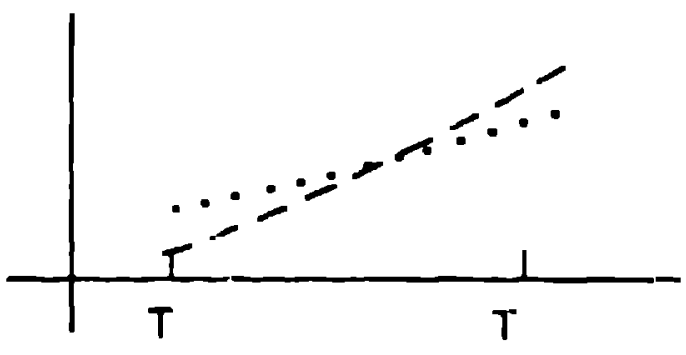

(c)

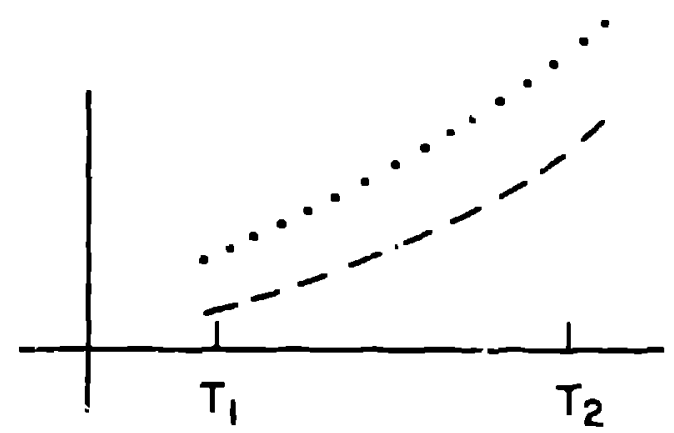

(b)

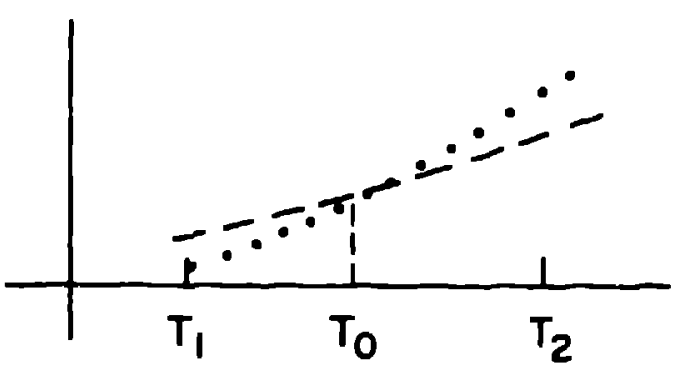

(d)

In case (a), only Fo is possible; in case (b), only. Fl; in cise (c), either Fl or F2, and in rase (d), neither F1 nor F2. This suggests there 
may be an interactive, nonsimple, flame type in case (d), since neither simple flame can exist. The only way both reactions can be significant in the burning process is for their H's to be the same. It is clear from the graph that this occurs at a value $T_{0}\left(T_{1}, T_{2}\right)$. We therefore postulate a flame burning at temperature $T_{0}$ in which both reactions iake part. This situation can be fit into the framework of Case II in Ser. 3. Such a flame would be governed by (17) with $U=\left[\begin{array}{l}\mathrm{T} \\ \mathrm{Y}\end{array}\right]$. The reduced problem would be $(33,32)$. More specifically variant (A) of that problem applies, since

$$
U_{+}=\left[\begin{array}{l}
T_{0} \\
0
\end{array}\right] \text { is given. }
$$

We now indicate the solution of that problem. First, it is clear that for some $\theta \in(0,1), T_{0}$ may be represented as $T_{0}=\theta T_{1}+(1-\theta) T_{2}$. Hence $T_{0}-T_{-}=\theta Y_{-} Q_{1}+(1-\theta) Y_{-} Q_{2}$ and since $K_{i}=\left[\begin{array}{c}Q_{i} \\ -1\end{array}\right]$, we have

$$
U_{+}-u_{-}=\alpha_{1} k_{1}+\alpha_{2} K_{2} \text {, }
$$

where $\alpha_{1}=\theta Y_{-}, \alpha_{2}=(1-\theta) Y_{-}$. The ratio $r$ is now determined from $(34)$, and hence $\bar{M}=a_{1} / \alpha_{1}$, and the profile $U(\bar{x})$ from (33a).

The consistency check now requires (24) to hold with $T_{+}$replaced by $T_{0}$. This equation can be written

$$
\bar{s} \ln r=\mathrm{H}_{2}\left(\mathrm{~T}_{0}\right)-\mathrm{H}_{1}\left(\mathrm{~T}_{0}\right)
$$

Since $T_{0}$ was chosen so the right side vanishes, there appears to be an inconsistenry. But the inconsistency is only superficial; the equation (38) simply says that the previous value of $\mathrm{T}_{0}$ should be adjusted (by an anount $O(\bar{E}))$ to obtain a more accurate value.

It should be remarked that the "mixed" flame constructed in casc (I) apparently also exists in case (c), because again, $\mathrm{I}_{\mathrm{i}}$ have a common value at $T_{0}$. But in that case, the mixed flame is unstable in a certain sense: if its Lemperature is perturbed by a smill amount, it will rvolve into one of the two simple flames. In case (d), this instability does not oicur. 


\section{Bibliography}

1. J. Buckmaster and G. S. S. Ludford 1982, Theory of Laminar Flames, Cambridge University Press, New York.

2. P. C. Fife 1982, Propagating fronts in reactive media, in: NonJinear Problems, Present and Future, A. Bishop, D. Campbell, B, Nicolaenko, eds., North-Holland.

3. P. Fife and B. Nicolaenko 1982, Two-redction flame propagation, in preparation.

4. A. Kapila and G. S. S. Ludford 1977, Tho-step sequential reactions for large activation energies, Combustion and Flame, 167-176.

5. A. Liñan 1971, A theoretical analysis of premixed flame propagalion with an isothermal chain reaction, Tech. Report, Inst. Nac. Tec. Aerospacial "Esteban Terradas", Madrid.

6. S. B. Margolis 1982, Lean flame propagation with competing chemical reactions, Combust. Sci. and Technol., to appear.

7. S. B. Margolis and K. J. Matkov'sky 1981, Flane propagation will multiple fuels, SlAM J. Appl. Mith., to appear.

8. S. B. Margolis and B. J. Matkowsky 1981, Flame propagation with a sequential reaction mechanism, SIAM J. Appl. Math., lo appear. 